\title{
O Papel da Telessaúde na Pandemia Covid-19: Uma Experiência Brasileira
}

\author{
The Role of Telehealth in the Covid-19 Pandemic: \\ A Brazilian Experience
}

Rodolfo Souza da Silva (https://orcid.org/0000-0001-6848-3385) 1,2

Carlos André Aita Schmtiz (https://orcid.org/0000-0002-9003-9704) ${ }^{2}$

Erno Harzheim (https://orcid.org/0000-0002-8919-7916) ${ }^{1}$

Cynthia Goulart Molina-Bastos (https://orcid.org/0000-0002-2504-2915) 1,2

Elise Botteselle de Oliveira (https://orcid.org/0000-0001-9552-3282) ${ }^{2}$

Rudi Roman (https://orcid.org/0000-0002-2663-4314) ${ }^{1,2}$

Roberto Nunes Umpierre (https://orcid.org/0000-0001-9841-5543) 1,2

Marcelo Rodrigues Gonçalves (https://orcid.org/0000-0001-8516-8547) 1,2

${ }^{1}$ Programa de PósGraduação em

Epidemiologia, Universidade Federal do Rio Grande do Sul (UFRGS). R. Ramiro Barcelos 2400, Campus Saúde. 90035-003 Porto Alegre RS Brasil. marcelorog@gmail.com ${ }^{2}$ TelessaúdeRS, UFRGS. Porto Alegre RS Brasil.

\begin{abstract}
SARS-CoV-2, the virus that causes Covid-19, is the third coronavirus to cause severe disease in humans and to spread globally in the past two decades. In this context, several national public health departments, including the Brazilian Ministry of Health, highlighted what was, until then, considered a support service to the health system: telehealth and telemedicine. We intend to present the actions carried out by a national telehealth service in Brazil, both as a Primary Health Care (PHC) support service to professionals and to patients, as well as discussing the potential to reorganize a health system. This is a prevalence study that summarizes the measures adopted by Brazilian Telehealth Center from the 9th to the 27th epidemiological weeks of 2020 to support the health services of the Brazilian Unified Health System (SUS). There was an increase of $76.8 \%$ in the demand for telephone teleconsultations during the evaluated period compared to the same period in 2019, with $28.8 \%$ of the entire demand arising from doubts related to Covid-19. The Covid-19 pandemic demanded a quick response, with the organization of materials about the disease, a new team to carry out telemonitoring and teleconsultation activities, in addition to the creation of a manual for teleconsultations in Primary Health Care.
\end{abstract}

Key words Telehealth, Telemedicine, Covid-19, Primary Health Care
Resumo OSARS-CoV-2, vírus causador da Covid-19, é o terceiro coronavírus a causar doença grave em humanos e que apresentou disseminação global nas duas últimas décadas. Nesse contexto, diversos departamentos nacionais de saúde pública, entre eles o Ministério da Saúde do Brasil, trouxeram destaque àquilo que era, até então, considerado um serviço de apoio ao sistema de saúde: a telessaúde e a telemedicina. Pretendemos apresentar as ações realizadas por um serviço nacional de telessaúde no Brasil, tanto no suporte aos profissionais de saúde da Atenção Primária à Saúde quanto a pacientes, além de discutir o potencial de reorganizar um sistema de saúde. Estudo de prevalência que sumariza as ações de telemedicina adotadas pelo TelessaúdeRS-UFRGS no período da $9^{a}$ à $27^{a}$ semana epidemiológica de 2020 para apoio aos serviços de saúde do Sistema Único de Saúde (SUS). Houve aumento de 76,8\% da demanda de teleconsultorias telefônicas no período avaliado em comparação com o mesmo período em 2019, sendo $28,8 \%$ dessa demanda total decorrente de dúvidas relacionada à Covid-19. A pandemia por Covid-19 demandou rápida resposta com a organização de materiais sobre a doença, uma nova equipe para execução das atividades de telemonitoramento e teleconsultas, além da elaboração de um manual para teleconsultas na Atenção Primária à Saúde.

Palavras-chave Telessaúde, Telemedicina, Covid-19, Atenção Primária à Saúde 


\section{Introdução}

O SARS-CoV-2, vírus causador da Doença por Coronavírus de 2019 (Covid-19), é o terceiro coronavírus a causar doença grave em humanos e que apresentou disseminação global nas duas últimas décadas. Como principais motivos da preocupação associada ao quadro agudo de infecção, encontramos uma pneumonia atípica e um estado de coagulação intravascular disseminada. Globalmente, a Covid-19 alcançou, em outubro de 2020, o número de mais de $40 \mathrm{mi}$ lhões de casos, 1.132.676 óbitos em dez meses ${ }^{1}$ e provocou grandes mudanças nas sociedades de todos os continentes ${ }^{2}$. A Organização Mundial da Saúde declarou o estado de pandemia pela doença causada por esse coronavírus em 13 de março de 2020. No Brasil, o Ministério da Saúde já havia declarado em 3 de fevereiro do mesmo ano Emergência em Saúde Pública de Importância Nacional, e em menos de cinco meses se tornou o novo epicentro da pandemia ${ }^{3}$.

Com uma média de mil casos diários desde a semana epidemiológica 22 , sem vacina ou tratamento efetivo existentes, medidas de distanciamento da população em geral e isolamento de casos e contatos aparecem como as principais estratégias para retardar a expansão da Covid-19 de forma que o sistema de saúde possa responder ao aumento de demanda por leitos de internação, em especial, aqueles em unidades de terapia intensiva ${ }^{4}$.

Como principal ponto de contato para as pessoas e para coordenar o cuidado dos pacientes, de maneira que possam permanecer monitorados a partir dos seus domicílios, observamos uma forte ênfase colocada na porta de acesso do sistema de saúde brasileiro - a Atenção Primária à Saúde. Nesse contexto, diversos departamentos nacionais de saúde pública, entre eles o Ministério da Saúde, trouxeram destaque àquilo que era, até então, considerado um serviço de apoio ao sistema de saúde, a telessaúde e a telemedicina. Entretanto, ambas avançam em uma direção muito mais ampla, a de tornar-se um metasserviço ${ }^{5}$, com uma atuação híbrida, no campo da assistência direta, repositório de dados, transição de cuidado e real ordenadora das pessoas dentro do sistema de saúde ${ }^{6}$. No Brasil, a Lei no 13.989 , de 13 de abril de 2020 estabelece a definição e autoriza o uso da telemedicina, enquanto durar a epidemia de Covid-197.

Localizado no extremo sul do Brasil, o núcleo de telessaúde técnico-científico do Rio Grande do Sul, vinculado à Universidade Federal do Rio
Grande do Sul (TelessaúdeRS-UFRGS), desenvolve há 13 anos ações múltiplas de suporte aos profissionais da atenção primária à saúde do SUS (Figura 1), tendo realizado até setembro de 2020, 211.512 teleconsultorias, 81.461 telediagnósticos (e.g., espirometrias, dermatologia, estomatologia e oftalmologia) e cerca de 460 mil ações de regulação de consultas encaminhadas para os serviços especializados, com uma evitação de 74,78\%. Em epidemias anteriores, como no caso do H1N1 (2009), Dengue e Zika (2015), o TelessaúdeRSUFRGS atuou de maneira ágil e coordenada, desenvolvendo expertise em organizar times de resposta rápida às emergências em saúde pública. Após as mudanças na legislação brasileira relacionadas ao exercício da telemedicina durante a pandemia, o TelessaudeRS-UFRGS passou a complementar sua atuação também em contato direto com as pessoas que buscam atendimento no sistema público de saúde brasileiro, com atividades de teleconsulta e telemonitoramento.

Pretende-se, neste artigo, apresentar as ações realizadas por um serviço nacional de telessaúde no Brasil, tanto no suporte aos profissionais de saúde da APS quanto a pacientes, além de discutir o potencial de reorganizar um sistema de saúde.

\section{Métodos}

Estudo de prevalência que sumariza as medidas adotadas pelo TelessaúdeRS-UFRGS no período compreendido da $9^{a}$ à $27^{a}$ semana epidemiológica de 2020 para apoio aos serviços de saúde do Sistema Único de Saúde em todo o país.

Por meio do banco de dados de teleconsultorias realizadas nesse período, foi realizada a análise descritiva do perfil dos profissionais de saúde solicitantes dessas teleconsultorias para dúvidas relacionadas à Covid-19. Somado a isso, os temas das teleconsultorias foram classificados em básicos (caso suspeito/definição de caso, dúvidas de gestão e/ou administrativas e uso de equipamentos de proteção individual - EPIs) ou avançados (afastamento/atestado/isolamento, testagem diagnóstica e tratamento), conforme proposto por Guyatt et al. ${ }^{8}$. O acesso ao site do TelessaúdeRS-UFRGS, assim como ao hotsite específico sobre Covid-19, foi avaliado e comparado à evolução do número de teleconsultorias por semana epidemiológica ao longo do mesmo período, tanto no ano de 2020 quanto ao ano anterior.

As variáveis utilizadas para a avaliação descritiva de demanda foram os tipos de perguntas 
Figura 1. Fluxo demonstrando a relação entre as ações de teleconsultoria, telediagnóstico e consulta remota do TelessaúdeRS-UFRGS no que tange as pessoas e a rede de atenção à saúde.

Fonte: Elaborado pelos autores.

realizadas pelos profissionais, médicos e enfermeiros, solicitantes; características das questões (básicas ou avançadas); e o perfil dos solicitantes (idade, sexo, profissão, tipo de serviço de saúde APS ou não-APS - e região do país). No que tange as ações de teleconsulta e telemonitoramento, foram abordadas aquelas realizadas de 8 de abril de 2020 até 29 de julho de 2020 .

Por se tratar de um estudo de prevalência com avaliação de todo o universo de solicitantes que realizaram consultorias telefônicas ao TelessaúdeRS-UFRGS, durante o período supracitado, não foram realizados cálculos amostrais. Para a análise descritiva foram utilizadas frequência absoluta e relativa, média, desvios-padrão e proporções.

O presente estudo é parte do projeto "Apoio Clínico para os Profissionais da Atenção Primária à Saúde através de Ferramentas de Telessaúde" e foi aprovado pelo Comitê de Ética em Pesquisa do Hospital de Clínicas de Porto Alegre.

\section{Resultados}

No período compreendido pelas semanas epidemiológicas $9^{\mathrm{a}}$ à $27^{\mathrm{a}}$ de 2020 foram realizadas 24.699 consultorias telefônicas pelo canal 0800, das quais 7.054 foram solicitadas por profissionais médicos ou enfermeiros e relacionadas à $\mathrm{Co}$ vid-19. Entre os médicos que solicitaram orientações sobre Covid-19, a maior prevalência foi do sexo feminino $(72,4 \%)$, com idade média de 38,2 anos, sendo a idade máxima de 82 anos e a mínima de 22 anos. A formação dos solicitantes em sua maioria era medicina $(62,5 \%)$, seguidos por enfermagem (36,9\%). Estes profissionais estavam alocados predominantemente na região $\mathrm{Su}$ deste $(36,81 \%)$ e seu local de trabalho era a APS $(57,4 \%)$. Ao avaliarmos a frequência de evitação de encaminhamentos, esta foi de $95,83 \%$. A Tabela 1 traz as os dados supracitados classificados entre profissionais de medicina e enfermagem.

Em relação ao tipo de dúvida dos profissionais de saúde sobre uma nova doença - a Covid-19 - (Figura 2), durante o período analisado a maior frequência esteve relacionada a questões avançadas do que questões básicas, sendo os profissionais médicos e enfermeiros os responsáveis por $99,4 \%$ das 7.099 questões apresentadas no período. A Figura 3 compreende as classificações de teleconsultorias mais frequentes compreendidas dentro dos tipos de dúvidas, sendo as categorias "caso suspeito/definição de caso", "gestão/ administrativo", "EPIs", "afastamento/atestado/ isolamento", "testagem diagnóstica" e "tratamento" responsáveis por $80 \%$ do total de questões.

No que tange a temporalidade, as questões consideradas básicas apresentaram maior número de solicitações no início da pandemia até a $13^{\mathrm{a}}$ semana epidemiológica, enquanto as questões avançadas aumentaram progressivamente ao longo da maior parte do período (Figura 3).

A demanda do canal 0800 de teleconsultorias do TelessaúdeRS-UFRGS, quando comparados os períodos compreendidos da $9^{\mathrm{a}}$ a $27^{\mathrm{a}}$ semanas 
Tabela 1. Características dos profissionais de saúde solicitantes e dos desfechos de teleconsultorias telefônicas do TelessaúdeRS-UFRGS em relação a desfecho e tipo de questão sobre COVID-19 no período compreendido entre a $9^{a}$ e $27^{a}$ semana epidemiológicas de 2020.

\begin{tabular}{|c|c|c|}
\hline Característica & Médicos & Enfermeiros \\
\hline \multicolumn{3}{|l|}{ Solicitantes } \\
\hline \multicolumn{3}{|l|}{ Idade (anos) } \\
\hline Média (DP) & $38,33(11,59)$ & $37,91(8,26)$ \\
\hline Mínima - Máxima & $23-82$ & $22-80$ \\
\hline \multicolumn{3}{|l|}{ Sexo } \\
\hline Masculino n (\%) & $1.675(37,77)$ & $268(10,23)$ \\
\hline Feminino n (\%) & $2.760(62,23)$ & $2.351(89,77)$ \\
\hline \multicolumn{3}{|l|}{ Tipo de serviço de saúde } \\
\hline APS n $(\%)$ & $2.865(64,60)$ & $1.185(45,25)$ \\
\hline Não-APS n (\%) & $1.570(35,40)$ & $1434(54,75)$ \\
\hline \multicolumn{3}{|l|}{ Região do país } \\
\hline Sudeste n (\%) & $1.507(33,98)$ & $1.093(41,73)$ \\
\hline Sul n (\%) & $1.503(33,89)$ & $692(26,42)$ \\
\hline Nordeste n (\%) & $821(18,51)$ & $433(16,53)$ \\
\hline Centro-Oeste n (\%) & $433(9,76)$ & $264(10,08)$ \\
\hline Norte n (\%) & $171(3,86)$ & $137(5,23)$ \\
\hline \multicolumn{3}{|l|}{ Teleconsultorias } \\
\hline \multicolumn{3}{|l|}{ Desfecho } \\
\hline Manter na APS n (\%) & $4.188(94,43)$ & $2.572(98,21)$ \\
\hline Encaminhar para atenção especializada n (\%) & $43(0,97)$ & $12(0,46)$ \\
\hline Encaminhar para rede de urgências e emergências n (\%) & $204(4,60)$ & $35(1,34)$ \\
\hline Total & 4.435 & 2.619 \\
\hline Questões básicas & $1.498(33,78)$ & $1.126(42,99)$ \\
\hline Caso suspeito/definição de caso n (\%) & $986(22,23)$ & $534(20,39)$ \\
\hline Gestão/administrativo n (\%) & $322(7,26)$ & $296(11,30)$ \\
\hline EPIs n (\%) & $190(4,28)$ & $296(11,30)$ \\
\hline Questões avançadas & $2.090(47,13)$ & $956(36,50)$ \\
\hline Afastamento/atestado/isolamento n (\%) & $1.269(28,61)$ & $627(23,94)$ \\
\hline Testagem diagnóstica n (\%) & $475(10,71)$ & $261(9,97)$ \\
\hline Tratamento $\mathrm{n}(\%)$ & $346(7,80)$ & $68(2,60)$ \\
\hline
\end{tabular}

epidemiológicas de 2019 e de 2020, apresentou um acréscimo de $76,8 \%$, sendo $28,8 \%$ dessa demanda decorrente da Covid-19 (Figura 4).

A Figura 5 mostra o aumento no número de acessos ao site do TelessaúdeRS-UFRGS, principalmente após a produção de conteúdo relacionado à Covid-19 (questões sumarizadas, manuais de conduta atualizados e materiais para pacientes). Esses acessos semanais passaram de cerca de 60 mil acessos por semana em 2019 para quase 120 mil acessos semanais em 2020.

As ações de teleconsulta e telemonitoramento desenvolvidas pelo TelessaúdeRS-UFRGS, em parceria com a Secretaria Municipal de Saúde de Porto Alegre e o Hospital de Clínicas de Porto
Alegre, no período de abril a julho de 2020, consistiram em 9.455 avaliações de pacientes provenientes de três fontes principais: a) internações prévias por doenças respiratórias crônicas (asma e doença pulmonar obstrutiva crônica), através de listas dos hospitais públicos do município; b) consultas dos ambulatórios de cardiologia HCPA (cardiopatia isquêmica e insuficiência cardíaca) que haviam sido suspensas devido a pandemia; e c) revisões de altas hospitalares pós-internação por Covid-19 e de casos suspeitos ou confirmados dos ambulatórios de APS e da emergência HCPA. Neste último grupo, composto por 3.951 avaliações, $151(2,7 \%)$ necessitaram avaliação presencial. Além dessas ações, foi desenvolvido 


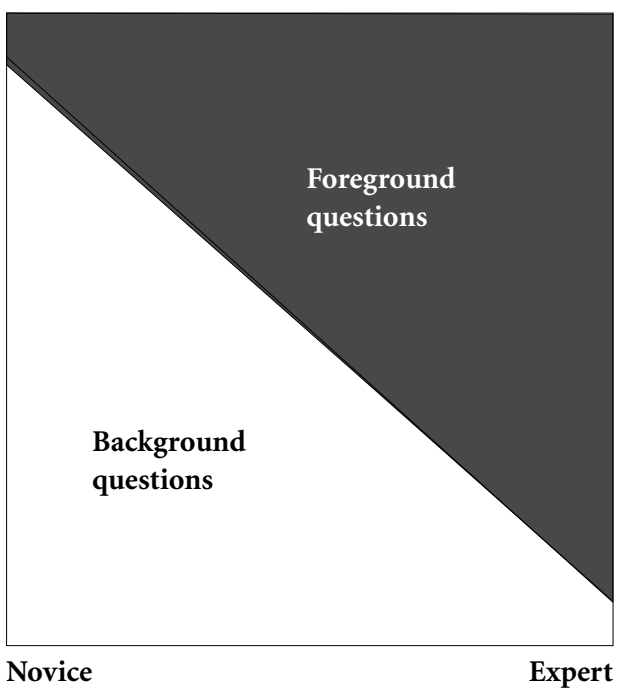

Figura 2. A evolução das dúvidas médicas sobre uma nova doença ao longo do tempo. (Foreground questions: dúvidas avançadas; Background questions: dúvidas básicas; Novice: médico recém-formado; Expert: médico experiente).

Fonte: Guyatt et al. ${ }^{8}$.

um manual para consulta remota, para auxílio aos profissionais de saúde de todo o Brasil a realizar essa nossa modalidade de consulta 9 .

\section{Discussão}

O presente estudo destaca as ações desenvolvidas por um serviço de telessaúde (Telessaúde RS-UFRGS) no enfrentamento a pandemia de Covid-19, tendo como resultado um aumento abrupto no número de teleconsultorias e a reorganização de um time de resposta rápida a pandemias, com produção de materiais sobre a nova doença, criação de serviços de telemonitoramento e teleconsulta, assim como a elaboração de um manual para teleconsulta na APS9.

Citada como o maior desafio sanitário do século XXI, a Covid-19 impactou o capital humano e financeiro de diversas instituições, com necessidades de adaptações inclusive dos serviços de saúde $^{10}$. Mesmo médicos experientes, assim como os com menor tempo de formação, necessitaram de informações consideradas tanto básicas, assim como as avançadas, sobre a nova condição clínica que se apresentou, e as ferramentas de apoio à tomada de decisão se apresentaram como mais essenciais do que nunca. O perfil dos solicitantes de teleconsultorias telefônicas relacionadas à Covid-19 foi composto majoritariamente por profissionais médicos e apresentou maior média de idade, acompanhando a tendência demonstrada pela demografia médica nacional, com a maior parte do sexo feminino ${ }^{11}$. No mesmo período, houve um aumento na frequência relativa e absoluta no número de teleconsultorias solicitadas por enfermeiras e enfermeiros. Em relação ao estabelecimento de saúde de atuação do profissional que entrou em contato com o TelessaúdeRS-UFRGS, houve expressivo aumento de outros estabelecimentos pela abertura de entrada de teleconsultorias pelo canal "Disque Saúde - 136" (hotline do Ministério da Saúde), demonstrando a necessidade de suporte a outros níveis de atenção à saúde além da APS, nicho de atuação do Programa Telessaúde Brasil Redes do qual o TelessaúdeRS-UFRGS faz parte. Até a 27a semana epidemiológica, nota-se também que a maior parte dos solicitantes eram da região sudeste, a mais populosa do país e a que concentrou o maior número absoluto de óbitos no país ${ }^{12}$.

Cabe destaque aos desfechos das teleconsultorias: há uma taxa de manutenção na APS de $95,83 \%$, superior inclusive a taxa apresentada nas teleconsultorias por outros temas, que no período foi de $72 \%$. Além da Lei de Roemer ${ }^{13}$, que destaca a importância da organização da demanda além do aumento do acesso, foi enfatizado o papel fundamental dos serviços de telessaúde durante a presente pandemia ${ }^{4,6,10,14,15}$. Diante da necessidade da qualificação da oferta ainda proposta por Roemer, condição sensível à telessaúde, devemos estar atentos às questões que são trazidas pelos solicitantes ${ }^{16}$. De acordo com o proposto por Guyatt et al. ${ }^{8}$ em relação ao aprendizado médico, as questões que normalmente são levantadas por um estudante de medicina devem ser consideradas como básicas, e a busca por questões que se seguem e, na maioria das situações, necessitam de compreensão das básicas para que façam sentido, são consideradas as avançadas. A evolução dessas dúvidas ao longo do tempo tende a ser progressiva, conforme ilustrado pela Figura 2.

Para compreender melhor o que se passou em relação ao serviço de teleconsultorias do TelessaúdeRS-UFRGS durante o início da aceleração da pandemia no Brasil, foram analisadas as teleconsultorias realizadas entre a $9^{a}$ e a $27^{a}$ semana epidemiológica de 2020. Observamos que 

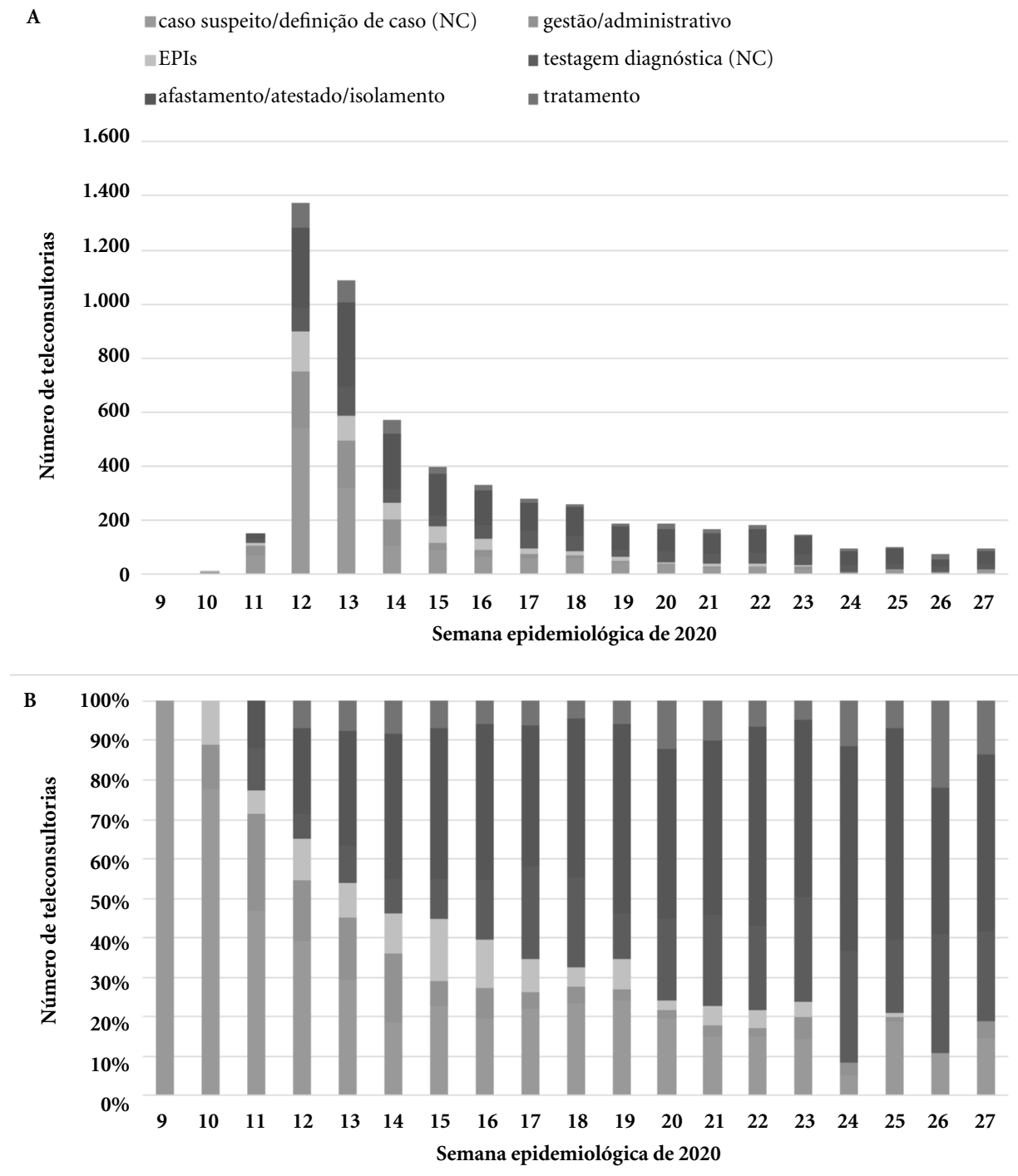

Figura 3. Evolução do tipo de dúvida dos profissionais de saúde sobre uma nova doença - a COVID-19 -, período compreendido pelas semanas epidemiológicas 9a e 27a de 2020. O Gráfico A apresenta a evolução da frequência absoluta de teleconsultorias, enquanto o Gráfico B demonstra essas teleconsultorias em frequências relativas.

Fonte: Elaborado pelos autores.

as teleconsultorias básicas relacionadas à Covid-19, as quais apresentaram como tema "caso suspeito/definição de caso", "gestão/administrativo" e "EPIs", foram as predominantes até a 13a semana epidemiológica (Figura 3-B). A partir da $14^{\mathrm{a}}$ semana epidemiológica, houve um aumento relativo (ainda que com decréscimo do número absoluto) progressivo do número de teleconsultorias relacionadas a temas que apresentaram desde o início da pandemia constante necessida- de de revisão, sendo eles "afastamento/atestado/ isolamento", "testagem diagnóstica" e "tratamento". Assim, de forma aplicada, vemos a teoria do aprendizado médico servindo como base para a preparação de materiais de referência para profissionais de saúde ${ }^{8}$.

Outro aspecto importante evidenciado neste estudo de caso é a necessidade de manter permanentemente equipes altamente qualificadas, com expertise em situações de emergência em saúde 


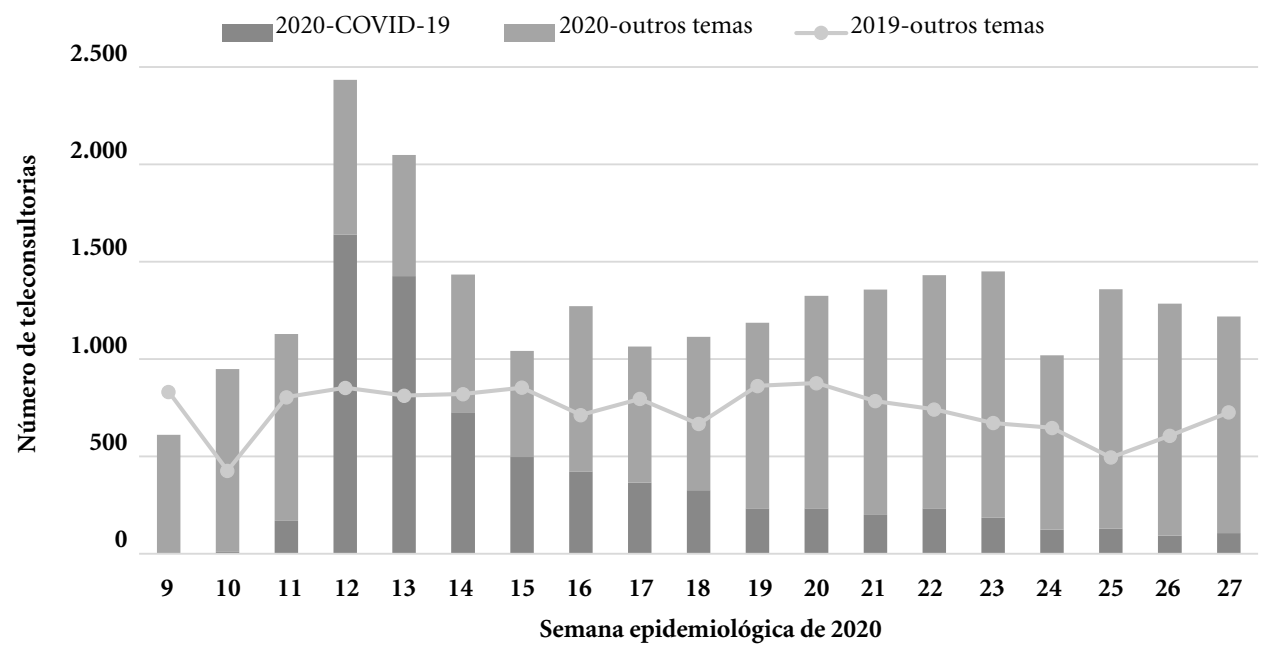

Figura 4. Comparação do número de teleconsultorias telefônicas do canal 0800 do TelessaúdeRS-UFRGS no período compreendido entre a 9a e a 27a semana epidemiológica de 2019 e 2020, sendo em 2020 divididas entre solicitações relacionadas à COVID-19 ou outros temas.

Fonte: Elaborado pelos autores.

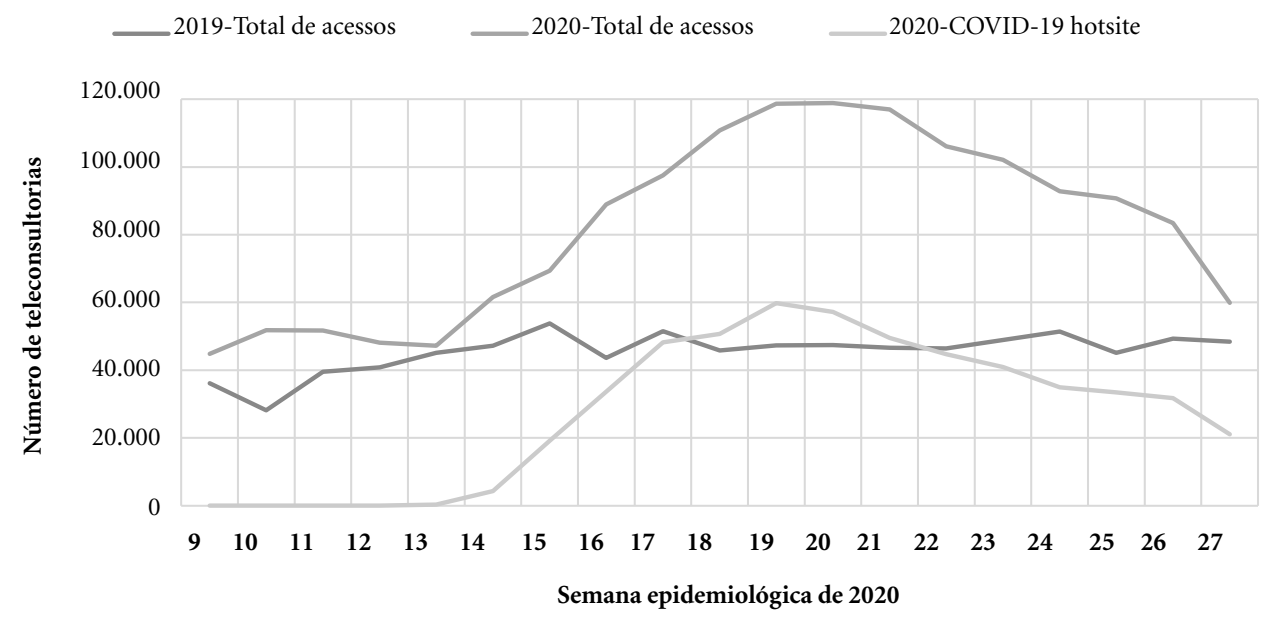

Figura 5. Comparação do número de acessos semanais ao hotsite COVID-19 em relação ao total de acessos semanais ao site do TelessaúdeRS-UFRGS entre a 9a e a $27^{a}$ semana epidemiológica em relação ao mesmo período de 2019.

Fonte: Elaborado pelos autores.

pública, como é o caso do TelessaúdeRS-UFRGS, que já havia participado intensamente em epidemias pregressas, como as de H1N1, Dengue e
$Z_{i k a}{ }^{17-20}$. Além do Time de Resposta Rápida, foi fundamental possuir infraestrutura adequada para o aumento de recursos humanos exigidos 
com a alta demanda, assim como apoio de tecnologia de informação e suporte governamental, através de agilidade na liberação e gestão de recursos. De maneira análoga, podemos comparar esta conformação aos depósitos secretos da Finlândia ${ }^{21,22}$, construídos na época da Guerra Fria, e utilizados durante a pandemia da Covid-19, minimizando o impacto da desaceleração econômica e escassez de produtos. Isso só foi viável devido a uma cultura de planejamento a longo prazo, prevenção de eventos catastróficos e flexibilidade frente aos desafios da contemporaneidade, os quais toda a humanidade foi lançada atualmente.

Por fim, partindo-se da necessidade de que as informações devem estar em todos os pontos de cuidado $^{23}$, embora já exista a tecnologia necessária para que os sistemas de informação sejam interoperáveis, ainda persistem obstáculos como a falta de uma cultura de compartilhamento de informações, os aspectos legais e normativos e a ineficiência administrativa ${ }^{24}$. Um grande serviço de telessaúde, como o apresentado neste artigo, com múltiplas tarefas, permite a racionalização no gasto dos recursos de saúde, ainda que esses recursos sejam insuficientes. Devem estar articuladas diferentes ações, como teleconsultoria, teleconsulta, telemonitoramento, telediagnóstico e teleducação, o que torna possível reduzir encaminhamentos desnecessários, utilização desnecessária de alta densidade tecnológica, além de reduzir a circulação física de pessoas, importante agora e na situação pós-Covid-19. Direcionar os pacientes dentro dos sistemas de saúde, verificando necessidades de acordo com a complexidade dos casos e preparando a APS, serviços de urgências e emergências, e hospitais para recebê-los será tarefa cada vez mais necessária, e a telessaúde aparece como importante protagonista dessa ação de coordenação dos diferentes agentes do sistema de saúde ${ }^{5}$.

\section{Colaboradores}

Concepção do artigo: RS Silva, CG Molina-Bastos, EB Oliveira, R Roman e MR Gonçalves. Desenho do artigo: RS Silva, CAA Schmtiz, E Harzheim, RN Umpierre e MR Gonçalves. Coleta dos dados: RS Silva, EB Oliveira e R Roman. Interpretação dos dados: RS Silva, CAA Schmtiz, CG Molina-Bastos, RN Umpierre e MR Gonçalves. Análise dos dados: RS Silva, CAA Schmtiz, E Harzheim e MR Gonçalves. Rascunho do artigo: RS Silva, CG Molina-Bastos, EB Oliveira, R Roman e MR Gonçalves. Revisão crítica: RS Silva, CAA Schmtiz, E Harzheim, RN Umpierre e MR Gonçalves. Todos os autores aprovaram a versão publicada, assim como acordam em ser responsáveis por todos os aspectos do trabalho para garantir que as questões relacionadas à precisão ou integridade de qualquer parte do trabalho sejam adequadamente investigadas e resolvidas. 


\section{Referências}

1. Johns Hopkins: University e Medicine. Coronavirus Resource Center. Global map [Internet]. Blatimore; 2020 [acessado 2020 out 23]. Disponível em: https:// coronavirus.jhu.edu/map.html.

2. Wiersinga WJ, Rhodes A, Cheng AC, Peacock SJ, Prescott HC. Pathophysiology, transmission, diagnosis, and treatment of coronavirus disease 2019 (COVID-19): a review. JAMA 2020; 324(8):782-793.

3. Brasil. Ministério da Saúde (MS). Portaria ${ }^{\circ} 188$, de 3 de fevereiro de 2020. Declara Emergência em Saúde Pública de importância Nacional (ESPIN) em decorrência da Infecção Humana pelo novo Coronavírus (2019-nCoV). Diário Oficial da União 2020; 4 fev.

4. Daumas RP, Silva GAE, Tasca R, Leite IC, Brasil P, Greco DB, Grabois V, Campos GWS. The role of primary care in the Brazilian healthcare system: limits and possibilities for fighting COVID-19. Cad Saude Publica 2020; 36(6): 00104120 .

5. Harzheim E, Chueiri PS, Umpierre RN, Gonçalves MR, Siqueira ACS, D'Avila OP, Molina-Bastos CG, Katz N, Dal Moro RG, Schmitz CAA. Telessaúde como eixo organizacional dos sistemas universais de saúde do século XXI. RBMFC 2019; 14(41):1-9.

6. Centers for Disease Control and Prevention (CDC). Health Healthcare Workers. Using telehealth to expand Access to Essential Health Services during the COVID-19 pandemic [Internet]. Georgia; 2020 [acessado 2020 out 23]. Disponível em: https://www.cdc.gov/ coronavirus/2019-ncov/hcp/telehealth.html.

7. Brasil. Ministério da Saúde (MS). Lei no 13.989 , de 15 de abril de 2020. Dispõe sobre o uso da telemedicina durante a crise causada pelo coronavírus (SARSCoV-2). Diário Oficial da União; 2020.

8. Guyatt G, Rennie D, Meade MO, Cook DJ. Users' guide to the medical literature. $3^{\mathrm{a}}$ ed. Nova York: McGraw -Hill; 2015.

9. Aita CAS, Gonçalves MR, Umpierre RN, Molina-Bastos CG, Silva RS. Manual de teleconsultas na APS. Porto Alegre: Artmed; 2021 [no prelo].

10. Harzheim E, Martins C, Wollmann L, Pedebos LA, Faller LA, Marques MC, Minei TSS, Cunha CRH, Telles LF, Moura LJN, Leal MH, Rodrigues AS, Rech MRA, D'Avila OP. Federal actions to support and strengthen local efforts to combat COVID-19: Primary Health Care (PHC) in the driver's seat. Cien Saude Colet 2020; 25(Supl. 1):2493-2497.

11. Associação Médica Brasileira. Demografia Médica 2018: número de médicos aumenta e persistem desigualdades de distribuição e problemas na assistência [Internet]. São Paulo; 2018 [acessado 2020 out 23]. Disponível em: https://amb.org.br/wp-content/uploads/2018/03/DEMOGRAFIA-M\%C3\%89DICA.pdf.

12. Instituto Militar de Engenharia (IME). Grupo de Assessoramento Científico do IME: grupo multidisciplinar. Sistemas de informações geográficas [Internet]. Rio de Janeiro: IME; 2020 [acessado 2020 out 23]. Disponível em: covid19científico.ime.eb.br.

13. Roemer MI. Bed supply and hospital utilization: a natural experiment. Hospitals 1961; 35:36-42.

14. Duckett $S$. What should primary care look like after the COVID-19 pandemic? Aust J Prim Health 2020; 26(3):207-211.
15. Krist AH, DeVoe JE, Cheng A, Ehrlich T, Jones SM. Redesigning Primary Care to address the COVID-19 pandemic in the midst of the pandemic. Ann Fam Med 2020; 18(4):349-354.

16. Katz N, Roman R, Rados DRV, Oliveira EB, Schmitz CAA, Gonçalves MR, Mengue SS, Umpierre RN. Acesso e regulação ao cuidado especializado no Rio Grande do Sul: a estratégia RegulaSUS do TelessaúdeRS-UFRGS. Cien Saude Colet 2020; 25(4):1389-1399.

17. Molina-Bastos CG, D'Avila OP, Umpierre RN, Faccini LS, Gonçalves MR, Harzheim E. Microcefalia e Zika Vírus: características e associações. Rev Bras Med Fam Comunidade 2016; 11(38):1-10.

18. Molina-Bastos CG, D'Avila OP, Umpierre RN, Gonçalves MR, Harzheim E. Zika Vírus. Rev Bras Med Fam Comunidade 2016; 11(38):1-3.

19. Universidade Federal do Rio Grande do Sul (UFRGS). Programa de Pós-Graduação em Epidemiologia. TelessaúdeRS (TelessaúdeRS-UFRGS). RS contra gripe [Internet]. [acessado 2020 out 23]. Disponível em: https://www.ufrgs.br/telessauders/gripe/.

20. Universidade Federal do Rio Grande do Sul (UFRGS). Centro Estadual de Vigilância Sanitária (Rio Grande do Sul). RS contra Aedes [Internet]. Porto Alegre; 2016-2017 [acessado 2020 out 23]. Disponível em: https://www.ufrgs.br/rscontraaedes/.

21. Stephen S, Issac A, Jacob K, Vijay VR, Radhakrishnan VR, Krishnan N. COVID-19: weighing the endeavors of nations, with time to event analysis. Osong Public Health Res Perspect. 2020; 11(4):149-157.

22. Anderson C, Libell HP. Finland, 'prepper Nation of the Nordics,' Isn't worried about masks [Internet]. The New York Times; 2020 [acessado 2020 out 23]. Disponível em: https://www.nytimes.com/2020/04/05/ world/europe/coronavirus-finland-masks.html.

23. Brasil. Ministério da Saúde (MS). Secretaria-Executiva. Departamento de Monitoramento e Avaliação do SUS. Política Nacional de Informação e Informática em Saúde. Brasília: MS; 2016.

24. Mello MM, Adler-Milstein J, Ding KL, Savage L. Legal barriers to the growth of Health Information Exchange-Boulders or Pebbles? Milbank Q 2018; 96(1):110143.

Artigo apresentado em 24/10/2020

Aprovado em 30/01/2021

Versão final apresentada em 02/02/2021

Editores-chefes: Maria Cecília de Souza Minayo, Romeu Gomes, Antônio Augusto Moura da Silva 
